\title{
CORRECTION
}

View Article Online

View Journal I View Issue

Check for updates

Cite this: Mater. Horiz., 2021, 8, 645

DOI: $10.1039 / \mathrm{d} 0 \mathrm{mh} 90085 \mathrm{k}$

rsc.li/materials-horizons

\section{Correction: Single nanosheet can sustainably generate oxygen and inhibit respiration simultaneously in cancer cells}

\author{
Wei-Qiang Huang, ${ }^{\text {ab }}$ Fei Wang, ${ }^{c}$ Ai-Zong Shen, ${ }^{\text {a }}$ Lei Zhang, ${ }^{a}$ Xuan Nie, ${ }^{b}$ \\ Ze Zhang, ${ }^{\text {b }}$ Guang Chen, ${ }^{\mathrm{b}}$ Lei Xia, ${ }^{\mathrm{b}}$ Long-Hai Wang, ${ }^{\mathrm{b}}$ Sheng-Gang Ding, ${ }^{\mathrm{d}}$ \\ Qing-Yong Meng, ${ }^{\text {e }}$ Wen-Jian Zhang, ${ }^{\text {b }}$ Chun-Yan Hong*b and Ye-Zi You*ab
}

Correction for 'Single nanosheet can sustainably generate oxygen and inhibit respiration simultaneously in cancer cells' by Wei-Qiang Huang et al., Mater. Horiz., 2021, DOI: 10.1039/d0mh01446j.

The authors regret errors in the affiliations listed in the originally published manuscript. The corrected list of authors and affiliations for this paper is as shown above.

The Royal Society of Chemistry apologises for these errors and any consequent inconvenience to authors and readers.

\footnotetext{
${ }^{a}$ The Department of Pharmacy, The First Affiliated Hospital of USTC, Division of Life Sciences and Medicine, University of Science and Technology of China, Hefei, Anhui, 230026, China.E-mail: yzyou@ustc.edu.cn,1649441800@qq.com

${ }^{b}$ Hefei National Laboratory for Physical Sciences at the Microscale, Hefei, Anhui, 230026, China. E-mail: hongcy@ustc.edu.cn, zze320@mail.ustc.edu.cn

${ }^{c}$ Neurosurgical Department, The First Affiliated Hospital of USTC, Division of Life Sciences and Medicine, Hefei, Anhui, 230026, China

${ }^{d}$ Anhui Medical University, Affiliated Hospital, Department of Pediatric, Hefei, Anhui, 230026, China. E-mail: dsg5312@163.com

${ }^{e}$ Anhui Medical University, Affiliated Hospital, Department of Radiotherapy, Anhui, People's Republic of China
} 